\title{
Quantification of Tryptophan Transport and Metabolism in Lung Tumors Using PET
}

Csaba Juhász ${ }^{1-4}$, Otto Muzik ${ }^{1,2,5}$, Xin Lu ${ }^{1}$, M. Salik Jahania ${ }^{4,6}$, Ayman O. Soubani ${ }^{4,7}$, Majid Khalaf ${ }^{1,2}$, Fangyu Peng ${ }^{1,2,4,5}$, Thomas J. Mangner ${ }^{1,5}$, Pulak K. Chakraborty ${ }^{1,5}$, and Diane C. Chugani ${ }^{1,2,5}$

${ }^{1}$ PET Center, Children's Hospital of Michigan, Wayne State University, Detroit, Michigan; ${ }^{2}$ Department of Pediatrics, Wayne State University School of Medicine, Detroit, Michigan; ${ }^{3}$ Department of Neurology, Wayne State University School of Medicine, Detroit, Michigan; ${ }^{4}$ The Barbara Ann Karmanos Cancer Institute, Wayne State University, Detroit, Michigan; ${ }^{5}$ Department of Radiology, Wayne State University School of Medicine, Detroit, Michigan; ${ }^{6}$ Department of Surgery, Wayne State University School of Medicine, Detroit, Michigan; and ${ }^{7}$ Department of Medicine, Wayne State University School of Medicine, Detroit, Michigan

\begin{abstract}
Abnormal tryptophan metabolism catalyzed by indoleamine 2,3dioxygenase may play a prominent role in tumor immunoresistance in many tumor types, including lung tumors. The goal of this study was to evaluate the in vivo kinetics of $\alpha-{ }^{11} \mathrm{C}$-methylL-tryptophan (AMT), a PET tracer for tryptophan metabolism, in human lung tumors. Methods: Tracer transport and metabolic rates were evaluated in 18 lesions of 10 patients using dynamic PET/CT with AMT. The kinetic values were compared between tumors and unaffected lung tissue, tested against a simplified analytic approach requiring no arterial blood sampling, and correlated with standardized uptake values (SUVs) obtained from ${ }^{18}$ F-FDG PET/CT scans. Results: Most non-small cell lung cancers (NSCLCs) showed prolonged retention of AMT, but 3 other lesions ( 2 benign lesions and a rectal cancer metastasis) and unaffected lung tissue showed no such retention. Transport and metabolic rates of AMT were substantially higher in NSCLCs than in the other tumors and unaffected lung tissue. A simplified analytic approach provided an excellent estimate of transport rates but only suboptimal approximation of tryptophan metabolic rates. ${ }^{18} \mathrm{~F}-\mathrm{FDG}$ SUVs showed a positive correlation with AMT uptake, suggesting higher tryptophan transport and metabolism in tumors with higher proliferation rates. Conclusion: Prolonged retention of AMT in NSCLCs suggests high metabolic rates of tryptophan in these tumors. AMT PET/CT may be a clinically useful molecular imaging method for personalized cancer treatment by identifying and monitoring patients who have increased tumor tryptophan metabolism and are potentially sensitive to immunopharmacotherapy with indoleamine 2,3dioxygenase inhibitors.
\end{abstract}

Key Words: lung tumors; positron emission tomography; tryptophan; metabolism; 2-deoxy-2-[18F]fluoro-D-glucose

J Nucl Med 2009; 50:356-363

DOI: 10.2967/jnumed.108.058776

$\mathbf{T}$ ryptophan is an essential amino acid required for biosynthesis of proteins, serotonin, and melatonin in the brain and other tissues (1). In mammals, however, most of

Received Oct. 3, 2008; revision accepted Nov. 21, 2008.

For correspondence or reprints contact: Csaba Juhász, PET Center,

Children's Hospital of Michigan, 3901 Beaubien Blvd., Detroit, MI 48201.

E-mail: juhasz@pet.wayne.edu

COPYRIGHT @ 2009 by the Society of Nuclear Medicine, Inc. the tryptophan derived from the diet is metabolized via the kynurenine pathway (2). Abnormal tryptophan oxidation along this pathway is an important mechanism for modulation of tumor cell proliferation and immunoresistance, mainly via the initial and rate-limiting step catalyzed by indoleamine 2,3-dioxygenase (IDO) $(3,4)$. Induction of IDO leads to local tryptophan depletion, thus inhibiting cell growth in some malignant tumors $(5,6)$. On the other hand, enhanced IDO activity in tumors may also exert a potent immunosuppressive effect by blocking T-lymphocyte proliferation, thus diminishing T-cell-mediated tumor rejection $(3,4,7)$. Thus, manipulation of tryptophan metabolism via the kynurenine pathway may have important implications in tumor pharmacotherapy. However, it is not always clear whether IDO activity should be enhanced or inhibited to suppress tumor growth in specific tumors.

Recent studies have consistently shown high expression of IDO in a variety of human tumors, including lung tumors $(3,8-14)$. Several of these studies demonstrated that high expression of IDO was associated with reduced survival (9$11,14)$. In vivo detection of abnormal tryptophan transport and metabolism via the kynurenine pathway could be an important tool in identifying patients amenable to immunotherapy targeting tryptophan metabolism in tumors and monitoring therapeutic effects. The PET radiotracer $\alpha-{ }^{11} \mathrm{C}$ methyl-L-tryptophan (AMT) is well suited for such studies; AMT is not a substrate for protein synthesis (15) but can be metabolized by IDO because of the low substrate specificity of this enzyme (16). Our previous studies detected a high concentration of quinolinic acid, a metabolite of the kynurenine pathway, in resected tubers showing increased AMT uptake on PET in children with tuberous sclerosis (17). In our recent studies of brain tumors, increased AMT uptake associated with expression of IDO was found in human gliomas and glioneuronal tumors on PET $(12,18)$.

The purpose of the present study was to explore the clinical use of AMT PET in extracerebral tumors. We selected thoracic tumors for this pilot study because lung tumors express IDO $(9,13)$; dynamic AMT PET of such 
tumors can conveniently include the heart in the field of view, thus allowing us to obtain arterial blood input function from the left ventricle of the heart for a full kinetic analysis; and lung tissue shows low background for AMT transport and metabolism. The overall goal of this study was to establish the feasibility of AMT PET for detecting altered tryptophan metabolism in extracerebral (primary and metastatic thoracic) tumors. Specific goals of the study were to explore whether AMT PET detects increased transport or metabolic rate in lung tumors, as compared with unaffected lung tissue, and whether different tumor types show different AMT transport or metabolic rates; determine whether estimation of AMT transport and metabolic rates could be achieved without arterial blood sampling, using a simplified graphical approach (19); and determine the relationship between AMT kinetic values and ${ }^{18}$ F-FDG uptake values in tumors from patients who underwent both ${ }^{18} \mathrm{~F}-\mathrm{FDG}$ and AMT PET.

\section{MATERIALS AND METHODS}

\section{Patients}

Ten patients ( 6 men; mean age, $55 \mathrm{y}$; Table 1$)$ with a diagnosis of a lung or mediastinal tumor underwent AMT PET/CT. The tumors were diagnosed by a clinical CT scan and surgical histology. Eight patients also underwent clinical ${ }^{18} \mathrm{~F}$-FDG PET/CT in our center. Of the 10 patients, 8 had a recent diagnosis of a lung or mediastinal tumor and 2 had an earlier diagnosis of lung cancer but showed progression after chemoradiotherapy (administered at least 6 mo before the AMT PET/CT scan). Tumor histology showed non-small cell lung cancer (NSCLC) in 7, a metastatic adenocarcinoma (from a colorectal cancer) in 1, and a benign lesion (hamartoma, thymic cyst) in 2 patients. Five patients with NSCLC had multiple tumors. AMT and ${ }^{18}$ F-FDG PET were performed within 1 mo (6-31 d; mean, $18 \mathrm{~d}$ ) on all 7 patients with a malignant tumor and within $9 \mathrm{wk}$ on the patient (patient 2) with the benign lung lesion. The study was approved by the Human Investigation Committee at Wayne State University, and written informed consent was obtained from each participant before the AMT PET/CT scan.

\section{PET Data Acquisition}

PET was performed using a Discovery STE PET/CT scanner (GE Healthcare). All data were acquired in high-resolution 3-dimensional mode and reconstructed using a 3-dimensional iterative algorithm.

The ${ }^{18} \mathrm{~F}$-FDG tracer was synthesized according to the method of Hamacher et al. (20). Patients fasted for at least $4 \mathrm{~h}$ before the ${ }^{18}$ F-FDG PET study, and the tracer (5.291 MBq/kg [0.143 mCi/ $\mathrm{kg}]$ ) was injected intravenously. After a 60-min uptake period, the patients were positioned supine in the PET/CT scanner, and a

\section{TABLE 1. Patient Data and SUVs Measured in Each Lesion}

\begin{tabular}{|c|c|c|c|c|c|c|c|c|}
\hline \multirow[b]{2}{*}{$\begin{array}{c}\text { Patient } \\
\text { no. }\end{array}$} & \multirow[b]{2}{*}{ Sex } & \multirow[b]{2}{*}{$\begin{array}{l}\text { Age } \\
\text { (y) }\end{array}$} & \multirow[b]{2}{*}{ Diagnosis } & \multirow{2}{*}{$\begin{array}{l}\text { Previous therapy } \\
\text { (before AMT) }\end{array}$} & \multirow{2}{*}{$\begin{array}{c}\text { Time between } \\
{ }^{18} \mathrm{~F}-\mathrm{FDG} \\
\text { and AMT PET }\end{array}$} & \multirow{2}{*}{$\begin{array}{l}\text { Lesion } \\
\text { no. }\end{array}$} & \multicolumn{2}{|c|}{ SUV } \\
\hline & & & & & & & ${ }^{18} \mathrm{~F}-\mathrm{FDG}$ & AMT \\
\hline 1 & $\mathrm{~F}$ & 54 & Thymic cyst & None & $\begin{array}{c}{ }^{18} \mathrm{~F}-\mathrm{FDG} \text { PET } \\
\text { not done }\end{array}$ & 1 & - & 0.5 \\
\hline 2 & M & 39 & Hamartoma & None & $54 \mathrm{~d}$ ( ${ }^{18} \mathrm{~F}-\mathrm{FDG}$ later) & 1 & 0.9 & 0.7 \\
\hline 3 & M & 66 & $\begin{array}{l}\text { Metastasis } \\
\quad \text { (rectal cancer) }\end{array}$ & None & $11 \mathrm{~d}$ & 1 & 2.8 & 1.3 \\
\hline 4 & M & 58 & NSCLC (scc) & $\begin{array}{l}\text { Chemotherapy } \\
(2 \mathrm{wk})^{\star}\end{array}$ & $1 \mathrm{mo}$ & 1 & 15.7 & 2.5 \\
\hline \multirow[t]{2}{*}{5} & $\mathrm{~F}$ & 58 & NSCLC (pd) & $\begin{array}{l}\text { Radiotherapy }+ \\
\text { chemotherapy } \\
(>6 \mathrm{mo})^{\dagger}\end{array}$ & $1 \mathrm{mo}$ & 1 & 8.0 & 5.5 \\
\hline & & & & & & 2 & 6.8 & 4.4 \\
\hline \multirow[t]{5}{*}{6} & $\mathrm{~F}$ & 56 & NSCLC (lcc) & $\begin{array}{l}\text { Radiotherapy }+ \\
\text { chemotherapy } \\
(>6 \mathrm{mo})^{\dagger}\end{array}$ & $7 d$ & 1 & 5.5 & 2.4 \\
\hline & & & & & & 2 & 2.8 & 2.7 \\
\hline & & & & & & 3 & 3 & 2.6 \\
\hline & & & & & & 4 & 2.7 & 1.6 \\
\hline & & & & & & 5 & 2.7 & 2.7 \\
\hline 7 & M & 79 & NSCLC (acc) & None & $6 \mathrm{~d}$ & 1 & 2.8 & 1.2 \\
\hline \multirow[t]{2}{*}{8} & M & 49 & NSCLC (scc) & None & $11 d$ & 1 & 7.3 & 2.1 \\
\hline & & & & & & 2 & 3.3 & 1.7 \\
\hline \multirow[t]{2}{*}{9} & $\mathrm{~F}$ & 47 & NSCLC (acc) & None & $\begin{array}{c}{ }^{18} \mathrm{~F}-\mathrm{FDG} \text { PET } \\
\text { not done }\end{array}$ & 1 & - & 3.2 \\
\hline & & & & & & 2 & - & 3.0 \\
\hline \multirow[t]{2}{*}{10} & M & 46 & NSCLC (pd) & None & $28 d$ & 1 & 4.7 & 1.9 \\
\hline & & & & & & 2 & 1.6 & 0.8 \\
\hline $\begin{array}{l}\text { *Therapy } \\
\text { †Therapy } \\
\text { scc }= \\
\text { Tumors }\end{array}$ & $\begin{array}{l}\text { ted } \\
\text { s fini } \\
\text { imou } \\
\text { con }\end{array}$ & $\begin{array}{l}{ }^{18} \mathrm{~F}- \\
\mathrm{mo} \\
\text { Il ca } \\
\text { itivel }\end{array}$ & $\begin{array}{l}\text { PET but } 2 \text { wk be } \\
\text { Ian } 6 \text { mo before } A \\
\text { mbered in patients }\end{array}$ & $\begin{array}{l}\text { AMT PET scan. } \\
\text { PET scan. } \\
\text { rentiated carcinon } \\
\text { th multiple lesions. }\end{array}$ & cc $=$ large cell carci & ; acc $=$ & nocarcin & \\
\hline
\end{tabular}


low-intensity scout CT scan (120 keV, $10 \mathrm{~mA}$ ) was acquired. The axial field of view for the scan, usually from the neck to the pelvis, was selected on the basis of the scout scan, and a low-dose helical CT scan (120 keV, $100 \mathrm{~mA}$ ) was acquired for attenuation correction and anatomic localization. At $60 \mathrm{~min}$ after injection, a wholebody ${ }^{18}$ F-FDG PET scan encompassing multiple bed positions (4 min/bed position) was acquired.

The AMT tracer was produced by a synthesis module designed and built in-house as previously described (21). Patients fasted for $6 \mathrm{~h}$ before the AMT PET studies, to ensure stable plasma tryptophan and large neutral amino acid levels during the study. Initially, a venous line was established for administration of the AMT tracer $(14.8 \mathrm{MBq}[0.4 \mathrm{mCi} / \mathrm{kg}])$. The patients were then positioned supine in the PET/CT scanner, and a low-intensity scout CT scan was acquired. On the basis of the location of lung nodules obtained from the previously acquired ${ }^{18} \mathrm{~F}-\mathrm{FDG}$ scan, 1 bed position was selected at the level of the lungs/myocardium, and a low-dose CT scan was acquired for attenuation correction. A 60 -min dynamic scan $(12 \times 10,3 \times 60,3 \times 300$, and $4 \times 600 \mathrm{~s})$ coinciding with the tracer injection was initiated. The reconstructed isotropic spatial resolution of the dynamic AMT study was approximately $7-\mathrm{mm}$ full width at half maximum.

\section{Image Data Processing and Analysis}

Noninvasive determination of the arterial blood input function using dynamic PET of the left ventricle was obtained using an approach developed previously $(22,23)$. In this approach, a small region of interest (ROI) at the center of the left ventricle was used to derive a time-activity curve, which represented the arterial blood input function. Lung tumors were visually identified by 1 experienced observer on the basis of CT and ${ }^{18} \mathrm{~F}-\mathrm{FDG}$ PET (where available) images in all planes showing the tumor. Regions were then drawn manually on the CT images and copied to all time frames of the dynamic sequence to obtain regional time-activity curves. Control regions of similar size were also drawn in apparently unaffected lung tissue, far from the tumor, on the contralateral side (for patients with unilateral tumors). Semiquantitative analysis of ${ }^{18} \mathrm{~F}$-FDG and summed AMT images (40-60 min after injection) was performed using the mean standardized uptake value (SUV). The SUV calculation relates tracer concentration in tissue to the dose injected and the patient's mass: SUV = tissue concentration in ROI $\left[\mathrm{kBq} / \mathrm{cm}^{3}\right] /$ injected dose per weight $[\mathrm{MBq} / \mathrm{kg}]$.

\section{Compartmental Modeling and Identifiability Analysis}

The tracer kinetics of AMT in tumor tissue were assessed using a 3-compartmental model (Fig. 1) characterized by the parameter vector $\mathrm{K}_{1}-\mathrm{k}_{4}$, blood volume, and shift parameter. The rate constant $\mathrm{K}_{1}(\mathrm{~mL} / \mathrm{g} / \mathrm{min})$ represents the forward and $\mathrm{k}_{2}\left(\mathrm{~min}^{-1}\right)$ represents the reverse combined transport of AMT across the blood vessel, interstitial space, and cell membrane into the cell cytoplasm, in which it comprises the free compartment. Irreversible enzymatic conversion of AMT to its metabolites and accumulation in the metabolic compartment are characterized by the metabolic rate constant $\mathrm{k}_{3}$ $\left(\min ^{-1}\right)$. Finally, the rate constant $\mathrm{k}_{4}\left(\mathrm{~min}^{-1}\right)$ characterizes the reverse transport of AMT metabolites across the cell interstitial space and blood vessel back into the blood pool (Fig. 1).

The structure of the linearized model in the neighborhood of the computed solution was used for analysis of parameter identifiability. A singular value decomposition of the parameter sensitivity matrix $\left(\mathrm{X}_{\mathrm{ij}}=\mathrm{df}_{\mathrm{i}} / \mathrm{dp}_{\mathrm{j}}\right.$, where $\mathrm{f}_{\mathrm{i}}\left(\mathrm{p}_{\mathrm{j}}\right)$ is the tissue model function at time $\mathrm{i}$ dependent on the parameter vector $\mathrm{p}_{\mathrm{j}}$ ) was computed, yielding the
FIGURE 1. Threecompartmental model for AMT kinetics in lung tumor, using first-order rate constants (17). Transport rate constant $\mathrm{K}_{1}$ and outflow rate constant $k_{2}$ describe exchange of AMT between vascular space $\left(\mathrm{C}_{\mathrm{P}}\right)$ and cell cytoplasm, in which it comprises free

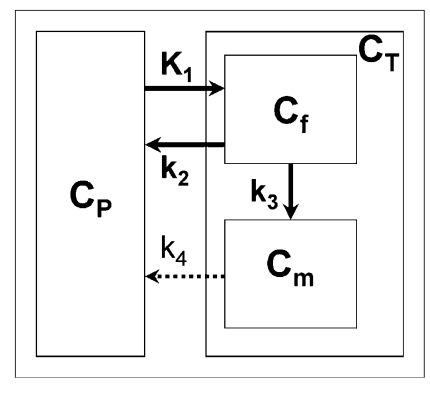
compartment $\left(\mathrm{C}_{\mathrm{f}}\right)$. Irreversible enzymatic conversion of AMT to its metabolites (in metabolic pool, $C_{m}$ ) is characterized by metabolic rate constant $k_{3}$. Efflux of AMT metabolites from metabolic compartment can be represented by rate constant $\mathrm{k}_{4}$. Although, theoretically, AMT metabolites could move from $C_{m}$ to both $C_{f}$ and $C_{P}$, applied kinetic modeling included efflux of tracer metabolites from entire tissue compartment. Dotted arrow represents $\mathrm{k}_{4}$, which was poorly identifiable in full model and eventually set to zero; thus, final analysis included only 3 kinetic rate constants $\left(\mathrm{K}_{1}\right.$, $\mathrm{k}_{2}$, and $\mathrm{k}_{3}$ ).

condition number $(\mathrm{CN})$ of the model (22). The $\mathrm{CN}$ of a model with orthonormal parameters is unity; hence, $\mathrm{CN}$ equals 1 in the ideal case. It was determined empirically (24) that weak dependencies are associated with $\mathrm{CNs}$ below 10, whereas strong dependencies are associated with CNs higher than 20.

\section{Simplified Analysis Using Patlak Graphical Method}

In the case of unidirectional uptake of the tracer into the metabolic compartment (i.e., for $\mathrm{k}_{4}=0$ ) and after dynamic equilibrium is reached, the unidirectional uptake rate constant $\mathrm{K}$ $\left(=\mathrm{K}_{1} \mathrm{k}_{3} /\left(\mathrm{k}_{2}+\mathrm{k}_{3}\right)\right)$ (units of $\left.\mathrm{mL} / \mathrm{g} / \mathrm{min}\right)$ can be determined using a linear approach first described by Patlak et al. (19),

$$
\frac{\mathrm{C}_{\mathrm{T}}(\mathrm{t})}{\mathrm{C}_{\mathrm{P}}(\mathrm{t})}=\mathrm{k}_{3} \mathrm{VD} \frac{\int_{0}^{t} \mathrm{C}_{\mathrm{P}}(\mathrm{u}) \mathrm{du}}{\mathrm{C}_{\mathrm{P}}(\mathrm{t})}+\varepsilon \mathrm{VD},
$$

with $\mathrm{C}_{\mathrm{T}}(\mathrm{t})$ representing tissue concentration derived from PET and $\mathrm{Cp}(\mathrm{t})$ representing the plasma tracer concentration. Furthermore, the rate constant $\mathrm{k}_{3}$ characterizes the enzymatic conversion of AMT, and $\operatorname{VD}\left(=\mathrm{K}_{1} /\left(\mathrm{k}_{2}+\mathrm{k}_{3}\right)\right)$ represents the volume of distribution of the tracer in the free precursor pool $\left(\mathrm{C}_{\mathrm{f}}\right)$. Finally, the factor $\varepsilon\left(=\mathrm{k}_{2} /\left(\mathrm{k}_{2}+\right.\right.$ $\mathrm{k}_{3}$ )) takes into account the time lag of the free precursor pool relative to the changing plasma tracer concentration. On reaching dynamic equilibrium $\left(\mathrm{dC}_{\mathrm{f}} / \mathrm{d}_{\mathrm{t}}=0 ; \mathrm{C}_{\mathrm{f}}(\mathrm{t})=\mathrm{VDC}(\mathrm{t})\right)$, Equation 1 describes a line with slope $\mathrm{k}_{3} \mathrm{VD}$ and intercept $\varepsilon \mathrm{VD}$. In case of irreversible uptake, the slope parameter $\left(\mathrm{k}_{3} \mathrm{VD}\right)$ corresponds to the previously described unidirectional uptake rate constant $\mathrm{K}$, and the intercept parameter equals $\varepsilon V D$. Finally, the ratio between slope and intercept allows estimation of the $\mathrm{k}_{3}$ parameter $\left(\mathrm{k}_{3}{ }^{\prime}=\mathrm{k}_{3} / \varepsilon\right)$.

\section{Statistical Analysis}

A multiple regression analysis was performed to establish which kinetic parameter was most influential for determining the unidirectional uptake rate constant K. Individual parameters were stepwise included, and the associated $r^{2}$ change in the linear model was used as a measure of importance for a particular parameter. Moreover, bivariate correlations between variables 
were assessed using the Pearson $r$ value. Statistical significance was assessed at a $P$ value of 0.05 .

\section{RESULTS}

\section{Identifiability of Compartmental Model Parameters}

Figure 2A shows a representative plane depicting tracer accumulation in NSCLC tissue of a patient (patient 6, 2NSCLC, Table 2), together with the corresponding timeactivity curves. The figure indicates good contrast between AMT tracer accumulation in tumor tissue and surrounding lung tissue. The slight decrease of tracer concentration in tumor tissue at late time points indicates the presence of reverse transport (efflux) of the AMT tracer from tumor tissue, which can be considered in the applied compartmental model through incorporation of a $\mathrm{k}_{4}$ parameter. Although application of the full 3-compartmental model
$\left(\mathrm{K}_{1}-\mathrm{k}_{4}\right)$ improved the data fit, it also resulted in poor identifiability of the overall parameter vector, rendering the obtained set of parameter values instable and highly dependent on noise. The average $\mathrm{CN}$ for the full 3compartmental model was determined as $22.6 \pm 6.9$ and decreased to $6.3 \pm 1.4$ when the $\mathrm{k}_{4}$ parameter was set explicitly to 0 . The reduced 3 -compartmental model $\left(\mathrm{K}_{1}-\right.$ $\mathrm{k}_{3}$ ) proved to be well conditioned, yielding robust estimates of the parameter vector. A detailed analysis of the 2 models showed a highly significant correlation among parameters $\mathrm{k}_{2}, \mathrm{k}_{3}$, and $\mathrm{k}_{4}(P<0.001$ for all bivariate correlations) in the full model, resulting in an overestimation of both $\mathrm{k}_{2}$ and $\mathrm{k}_{3}$ parameters. In contrast, in the reduced model, no significant correlation was determined between the rate constants $\mathrm{k}_{2}$ and $\mathrm{k}_{3}(P=0.54), \mathrm{K}_{1}$ and $\mathrm{k}_{3}$ $(P=0.12)$, or $\mathrm{K}_{1}$ and $\mathrm{k}_{2}(P=0.06)$. On the basis of
A
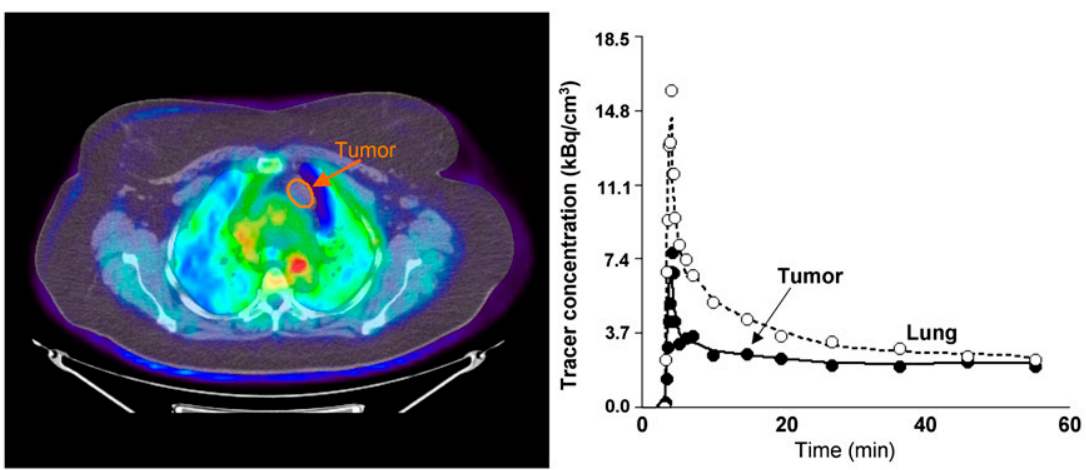

B
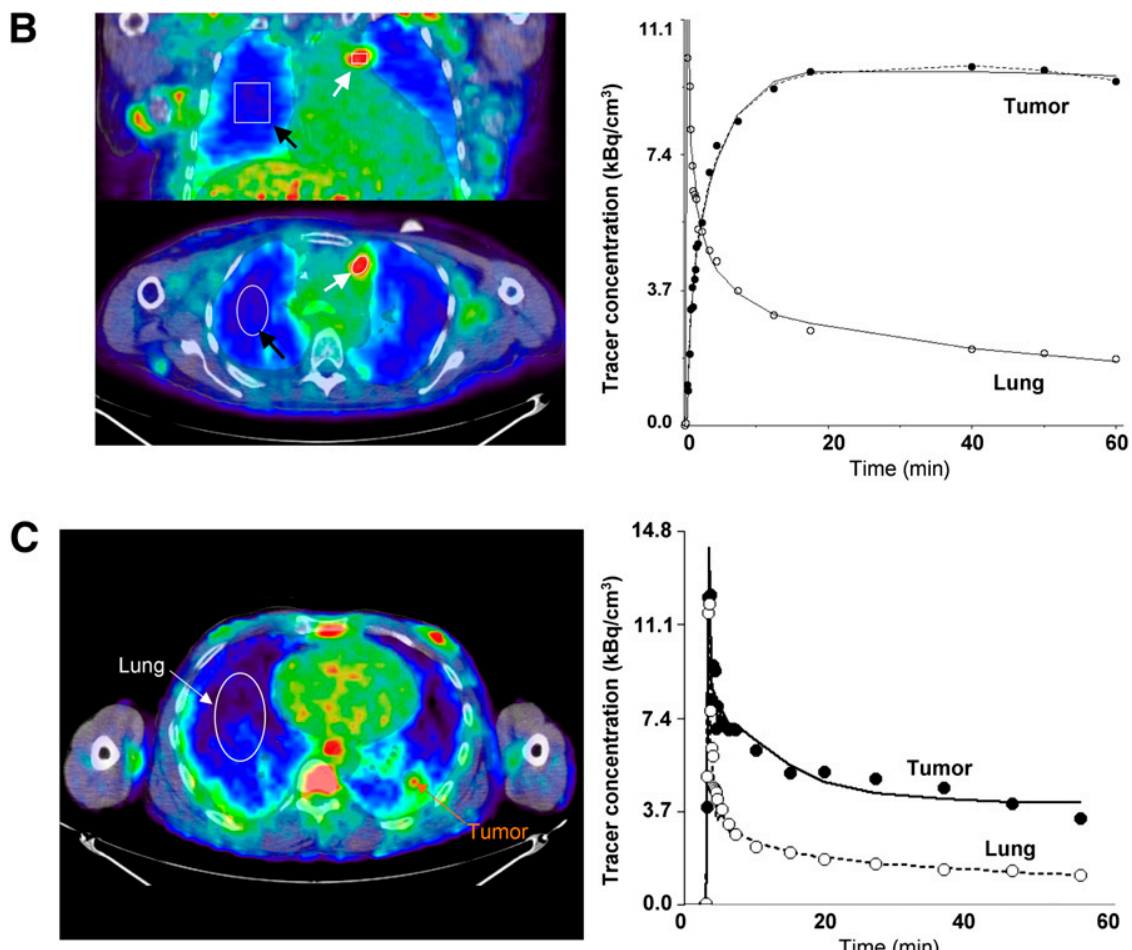

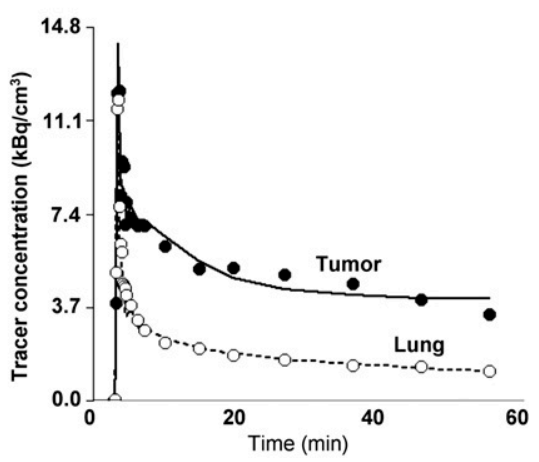

FIGURE 2. (A) Images of AMT tracer uptake between 40 and 60 min after injection (left) in patient with benign cyst (patient 1, Table 2). There is low accumulation of tracer in cyst, with activity being lower than normal lung tissue at all time points. (B) Images of AMT tracer uptake between 40 and 60 min after injection (left) in patient with NSCLC with multiple local metastases (patient 6, Table 2). Images show high accumulation in tumor tissue (2NSCLC, Table 2) and excellent contrast between tumor and lung tissue. ROls were defined for tumor nodules (white arrows) and lung tissue (black arrows). Corresponding tumor timeactivity curve (right) indicates rapid initial uptake of AMT, followed by slight decrease at late time points. Curve fit applying reduced compartmental model $\left(\mathrm{k}_{4}=0\right)$ is shown as solid line, and full compartmental model is displayed as broken line. In addition, 2-compartmental model fit is shown for lung tissue. (C) Representative image of AMT tracer uptake between 40 and 60 min after injection (left) in patient (patient 10, 2-NSCLC, Table 2). Two tumor sites were identified in this patient on basis of $\mathrm{CT}$ and ${ }^{18} \mathrm{~F}-\mathrm{FDG}$, which both were believed to represent NSCLC. First tumor (1-NSCLC, Table 2) showed time-activity curves similar to Figure $2 \mathrm{~B}$ and high ${ }^{18} \mathrm{~F}-\mathrm{FDG}$ SUV. In contrast, second site (orange arrow) showed relatively low uptake. Inspection of corresponding time-activity curve showed high initial uptake, with subsequent plateau followed by tracer washout. Neither full nor reduced (shown) 3-compartmental model fit data well; however, parameter identifiability was much improved using reduced model (CN 5.3 vs. 21.8). 


\section{TABLE 2. Individual Parameter Estimates Derived from Reduced Compartmental Model}

\begin{tabular}{|c|c|c|c|c|c|c|}
\hline Patient no. & Lesion & $\mathrm{K} 1$ (mL/g/min) & $\mathrm{k} 2\left(\mathrm{~min}^{-1}\right)$ & k3 $\left(\min ^{-1}\right)$ & $\mathrm{K}(\mathrm{mL} / \mathrm{g} / \mathrm{min})$ & Patlak slope \\
\hline 1 & Thymic cyst & 0.012 & 0.092 & 0.019 & 0.0021 & 0.0017 \\
\hline 2 & Hamartoma & 0.098 & 0.292 & 0.006 & 0.0019 & 0.0016 \\
\hline 3 & Metastasis & 0.098 & 0.279 & 0.006 & 0.0020 & 0.0007 \\
\hline 4 & NSCLC $^{\star}$ & 0.170 & 0.179 & 0.008 & 0.0070 & 0.0051 \\
\hline \multirow[t]{2}{*}{5} & 1-NSCLC & 0.287 & 0.195 & 0.017 & 0.0236 & 0.0108 \\
\hline & 2-NSCLC & 0.187 & 0.170 & 0.022 & 0.0213 & 0.0074 \\
\hline \multirow[t]{5}{*}{6} & 1-NSCLC & 0.073 & 0.067 & 0.01 & 0.0096 & 0.0088 \\
\hline & 2-NSCLC & 0.128 & 0.131 & 0.015 & 0.0129 & 0.0107 \\
\hline & 3-NSCLC & 0.104 & 0.105 & 0.014 & 0.0125 & 0.0095 \\
\hline & 4-NSCLC & 0.064 & 0.117 & 0.017 & 0.0082 & 0.0043 \\
\hline & 5-NSCLC & 0.063 & 0.036 & 0.006 & 0.0092 & 0.0035 \\
\hline 7 & $\mathrm{NSCLC}^{+}$ & 0.082 & 0.210 & 0.008 & 0.0031 & 0.0030 \\
\hline \multirow[t]{2}{*}{8} & 1-NSCLC & 0.176 & 0.131 & 0.011 & 0.0134 & 0.0074 \\
\hline & 2-NSCLC* & 0.275 & 0.211 & 0.004 & 0.0053 & 0.0058 \\
\hline \multirow[t]{2}{*}{9} & 1-NSCLC & 0.242 & 0.213 & 0.037 & 0.0359 & 0.0180 \\
\hline & 2-NSCLC & 0.184 & 0.151 & 0.026 & 0.0266 & 0.0152 \\
\hline \multirow[t]{2}{*}{10} & 1-NSCLC & 0.114 & 0.070 & 0.011 & 0.0145 & 0.0078 \\
\hline & 2-NSCLC* & 0.151 & 0.201 & 0.01 & 0.0075 & 0.0034 \\
\hline Total Mean & NSCLC & 0.153 & 0.146 & 0.014 & 0.0140 & 0.0081 \\
\hline Total SD & & 0.073 & 0.058 & 0.008 & 0.0091 & 0.0043 \\
\hline Mean for unaffected lungs & & 0.036 & 0.208 & - & - & - \\
\hline SD for unaffected lungs & & 0.014 & 0.047 & - & - & - \\
\hline
\end{tabular}

this identifiability analysis, the reduced 3-compartmental model $\left(\mathrm{K}_{1}-\mathrm{k}_{3}\right)$ was chosen for analysis of all tumor tissue time-activity curves.

\section{AMT Kinetics in Lung Tumors}

Individual rate constants $\left(\mathrm{K}_{1}, \mathrm{k}_{2}\right.$, and $\left.\mathrm{k}_{3}\right)$ were fitted to dynamic time-activity curves obtained from tumor tissue, and the unidirectional uptake rate for AMT $(\mathrm{K})$ was calculated (Table 2). AMT tracer accumulation in non-NSCLC tumors (patients 1-3, Table 1) was lower than that in lung tissue (Fig. 2A), yielding low K values (0.0019-0.0021 mL/ $\mathrm{g} / \mathrm{min}$ ) for these lesions (Table 2). This value was more than 10 times lower than values obtained for some of the NSCLC tumors with the highest uptake values.

In NSCLC tumors, 2 main types of time-activity curves were observed. Most NSCLCs showed continuous accumulation of the tracer (Fig. 2B); however, in a few NSCLCs (patient 4; patient 8, 2-NSCLC; and patient 10, 2-NSCLC) an initial accumulation phase with subsequent washout (Fig. 2C) was observed, resulting in intermediate $\mathrm{K}$ values (Table 2). In 1 patient with NSCLC (patient 7), the calculated tracer uptake was also relatively low, although the shape of the curve was similar to that observed in other NSCLC patients with high washout. The likely cause for the observed low tracer uptake may be partial-volume effects due to the small size of the tumor $\left(\sim 0.8 \mathrm{~cm}^{3}\right)$ as measured on the CT image. The average size of all other NSCLC tumors was $3.1 \pm 1.6 \mathrm{~cm}^{3}$ (range, $\left.1.2-7.3 \mathrm{~cm}^{3}\right)$.
A multiple regression analysis including $K_{1}$ and $k_{3}$ as predictors and the unidirectional uptake rate constant $(\mathrm{K})$ as outcome showed the $\mathrm{k}_{3}$ parameter to be the main predictor of $\mathrm{K}$ ( $r^{2}$ change was 0.55 for $\mathrm{k}_{3}$ but only 0.05 for $\mathrm{K}_{1}$ ). Consequently, $\mathrm{K}$ derived from the reduced compartmental model showed a highly significant correlation with the $\mathrm{k}_{3}$ parameter $(P<0.001$, Fig. $3 \mathrm{~A})$ and a significant but less prominent correlation with the $\mathrm{K}_{1}$ parameter $(P=$ 0.01 ), indicating that accumulation of the AMT tracer in tumor tissue is primarily due to metabolic conversion. In addition, a significant correlation was determined between the unidirectional uptake rate $(\mathrm{K})$ and the SUV for AMT $(P<0.001$, Fig. 3B $)$, suggesting that a semiquantitative measure can be used to estimate metabolic conversion of AMT.

\section{AMT Kinetics in Unaffected Lung Tissue}

Visual inspection of lung tissue time-activity curves showed continuous washout of AMT tracer (Figs. 2A-2C) in most patients, with little evidence of metabolic retention (except for 1 patient). On the basis of this observation, lung tissue timeactivity curves were fitted with a 2 -compartmental model $\left(\mathrm{K}_{1}\right.$, $\mathrm{k}_{2}$ ), with parameter $\mathrm{k}_{3}$ set explicitly to 0 . The 2-compartmental model fitted the curves well and yielded an excellent identifiability of the parameter vector $(\mathrm{CN}=3.6 \pm 0.3)$.

The $\mathrm{K}_{1}$ parameter characterizing unidirectional transport of AMT into tissue was found to be significantly lower in lung tissue $(0.037 \pm 0.013 \mathrm{~mL} / \mathrm{g} / \mathrm{min})$ than in NSCLC tumors $(0.153 \pm 0.074 \mathrm{~mL} / \mathrm{g} / \mathrm{min}, P=0.001)$. Moreover, both these values were higher than previously 

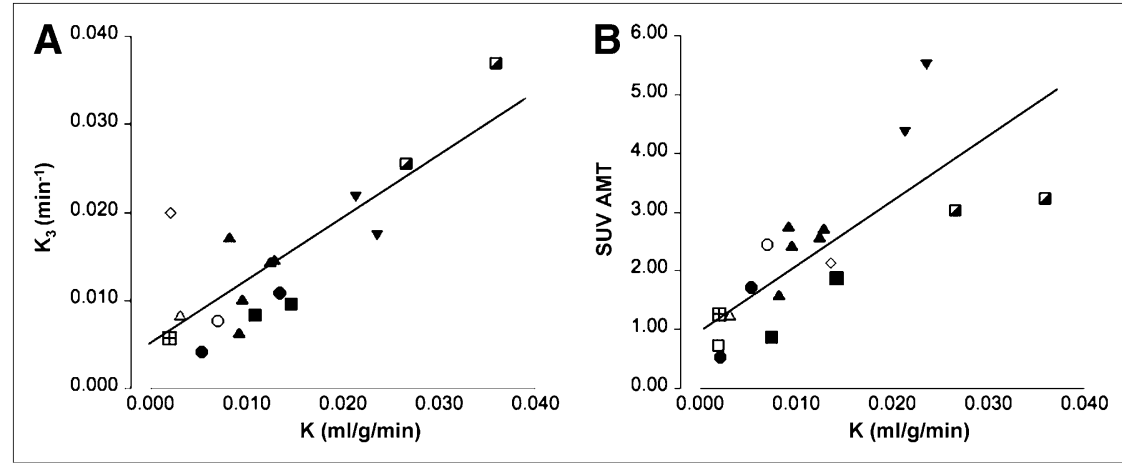

FIGURE 3. Correlation between unidirectional uptake rate $(K)$ derived from reduced compartmental model $\left(k_{4}=0\right)$ and either $k_{3}$ parameter (A) or SUV for AMT (B). Tumors obtained from different patients are marked by different symbols. $\mathrm{k}_{3}$ parameter was highly correlated with $\mathrm{K}$, indicating that metabolic conversion is dominant factor in AMT tracer uptake in tumors. Moreover, $\mathrm{K}$ was also significantly correlated with SUV for AMT, suggesting that this semiquantitative value can provide estimate of magnitude of metabolic conversion. determined in normal brain tissue $(0.014 \pm 0.003)(25)$, consistent with a lack of a blood-tissue barrier, such as the blood-brain barrier in the brain. In 1 patient (patient 5 ), visual inspection of lung tissue time-activity curves suggested possible metabolic retention of AMT; thus, we applied both a 2- and a 3-compartmental model to this patient's lung tissue curve. The $\mathrm{CN}$ (4.6 vs. 5.3 ) and the $\mathrm{K}_{1}$ parameter $(0.071 \mathrm{vs} .0 .076 \mathrm{~mL} / \mathrm{g} / \mathrm{min})$ for both models were similar, with a lower $\mathrm{k}_{2}$ parameter determined for the 2-compartmental model ( 0.271 vs. $\left.0.367 \mathrm{~min}^{-1}\right)$ and a relatively large value for the $\mathrm{k}_{3}$ parameter $\left(0.010 \mathrm{~min}^{-1}\right)$, resulting in a $\mathrm{K}$ value similar to that obtained in non-NSCLC tumors $(0.0021 \mathrm{~mL} / \mathrm{g} / \mathrm{min})$.

\section{Simplified Analysis of Kinetic Data}

In the absence of arterial blood sampling, complete timeactivity curves for the blood input function and the lung tumor can be obtained only when both the left ventricle of the heart and the tumor are in the field of view of the scanner. To determine whether a simplified analysis not requiring arterial sampling during the initial tracer uptake period could be used to determine $\mathrm{K}$ values, we applied the Patlak graphical analysis approach to data from 40 to $60 \mathrm{~min}$ after injection. The comparison of $\mathrm{K}$ values in tumors obtained from the reduced compartmental model and those derived from the simplified Patlak analysis showed an excellent correlation ( $r=0.83, P=0.001$, Fig. 4A), although $\mathrm{K}$ values derived using the Patlak graphical analysis were biased toward lower values. The $\varepsilon$-factor equal to the expression $\mathrm{k}_{2} /\left(\mathrm{k}_{2}+\mathrm{k}_{3}\right)$ derived from compartmental modeling was determined as $0.97 \pm 0.01$ for lung tissue and $0.92 \pm 0.05$ for tumor tissue. As this factor is close to unity, the volume of distribution (VD) of AMT in tissue can be approximated by the intercept derived from the Patlak graphical analysis. Figure 4B shows the correlation between VD values derived from compartmental analysis $\left(=\mathrm{K}_{1} /\left(\mathrm{k}_{2}+\mathrm{k}_{3}\right)\right)$ and the Patlak intercept. Although absolute values of VD were slightly overestimated using the Patlak approach, we found an excellent correlation between these 2 measures $(r=0.77, P<0.001)$.

The $\mathrm{k}_{3}$ parameter can also be estimated from the Patlak graphical analysis as the quotient between the slope and intercept of the linear fit. After removal of a highly influential point, the correlation between the $\mathrm{k}_{3}$ parameter derived from the reduced compartmental fit and that calculated from the Patlak graphical analysis was found to be not significant $(r=$ $0.46, P=0.07)$. The lack of correlation is likely the result of uncertainty associated with the quotient of the slope and intercept parameters.

\section{Comparison Between AMT and ${ }^{18} \mathrm{~F}-F D G$ SUVs}

We found a significant correlation between the ${ }^{18} \mathrm{~F}-\mathrm{FDG}$ and AMT SUVs $(r=0.73, P=0.004)$, with the AMT SUV being in general about half of the ${ }^{18} \mathrm{~F}-\mathrm{FDG}$ SUV. Moreover, the ${ }^{18} \mathrm{~F}$-FDG SUVs were highly correlated with $\mathrm{K}$ for AMT $(P=0.001)$ and to a lesser degree with both the $\mathrm{K}_{1}(P=0.02)$ and the $\mathrm{k}_{3}(P=0.04)$ parameters. A multiple regression analysis showed that only $\mathrm{K}$ for AMT was a significant independent predictor of ${ }^{18} \mathrm{~F}-\mathrm{FDG}$ SUV.
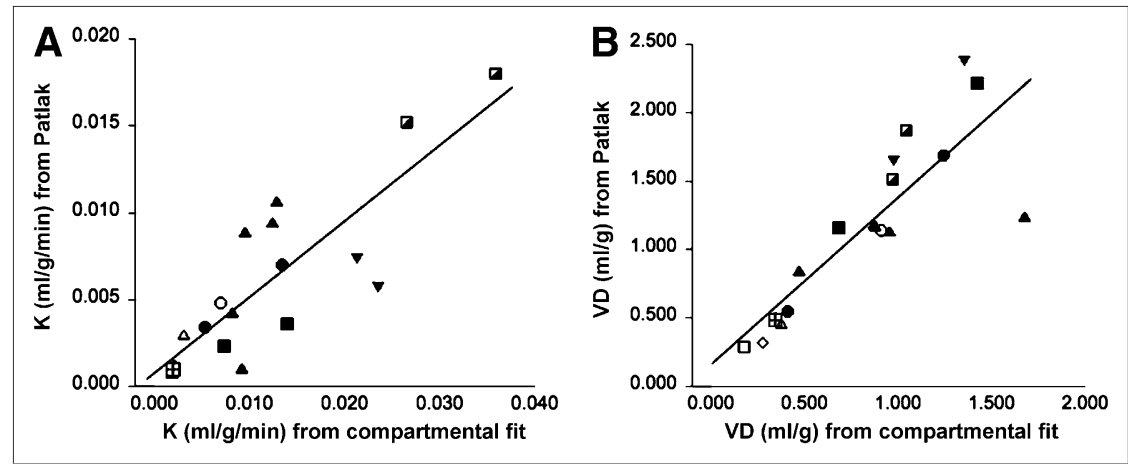

FIGURE 4. (A) Correlation of $K$ values derived from reduced compartmental fit and Patlak graphical analysis. $r$ was determined as 0.83 , indicating an excellent correlation between these 2 measures. Tumors obtained from different patients are marked by different symbols. (B) Excellent correlation was also determined between VD determined using compartmental fit and Patlak graphical analysis. 


\section{DISCUSSION}

This study demonstrates the feasibility of quantifying transport and metabolic rates of tryptophan tracer AMT in lung tumors. We have also shown preliminary evidence for higher unidirectional uptake of this PET tracer in NSCLCs, as compared with nontumorous lung tissue and non-NSCLC tumors, including benign lesions. Because AMT can be metabolized by IDO via the kynurenine pathway, it is likely that high AMT metabolic rates reflect increased tryptophan metabolism by this pathway in NSCLCs, although the exact metabolic rates for tryptophan itself cannot be calculated as the correction factor (lumped constant) between AMT and tryptophan is not known. A simplified analysis of the kinetic data, using the Patlak graphical approach with venous blood sampling, showed that although the unidirectional uptake rate constant $(\mathrm{K})$ and the tissue tracer VD are accurately estimated by the Patlak approach, evaluation of metabolic conversion alone (characterized by the $\mathrm{k}_{3}$ parameter) is suboptimal, especially in the middle of the value range. As a result, compartmental modeling is highly desirable if an accurate measurement of AMT metabolic rates is required. This AMT PET study in lung tumors suggests that this imaging method may also be useful in other extracerebral tumors with abnormal tryptophan metabolism.

Our study demonstrates a positive correlation between ${ }^{18} \mathrm{~F}-\mathrm{FDG}$ and AMT SUVs, although ${ }^{18} \mathrm{~F}-\mathrm{FDG}$ SUV was best predicted by the unidirectional uptake rate constant. The ${ }^{18} \mathrm{~F}$-FDG SUV is a strong predictor of proliferative activity in NSCLCs $(26,27)$. The relationship between ${ }^{18} \mathrm{~F}$ FDG SUV and AMT kinetic values suggests that lung tumors with higher proliferative activity have higher transport rates and higher metabolism of tryptophan, likely via the kynurenine pathway. High AMT transport could be due to increased vascularity and the presence of newly formed vessels with increased permeability. A similar effect was seen in malignant gliomas, in which signs of an impaired blood-brain barrier were associated with high VD values for AMT (18). High AMT transport can also reflect an upregulation of the transport system that supplies tryptophan for protein synthesis and also for IDO. Tryptophan influx into cells is supported by the plasma membrane transport system L, which is responsible for the transport of large neutral amino acids including several essential amino acids such as tryptophan (28). The neutral L-type amino acid transporter 1 (LAT-1) (29) is highly expressed in almost all studied tumor cell lines and tumor types, playing an important role in allowing cell growth $(30,31)$. A recent study showed in a large cohort $(n=321)$ of patients with NSCLC that expression of LAT-1 has a strong prognostic value for metastatic disease and the 5-y survival rate (32). Interestingly, 1-methyl-tryptophan, a potent competitive inhibitor of IDO, which is being tested as an anticancer agent to break tumor immunoresistance, is also an inhibitor of L-tryptophan transport (33). AMT PET could identify tumors with high LAT-1 expression and high IDO activity and also monitor the effect of IDO inhibitors on tryptophan transport and metabolism.

The findings of the current study show some intriguing differences between lung tumors and brain tumors in terms of AMT kinetics. Our recent studies in brain tumors have demonstrated higher transport rates but lower metabolic rates of AMT in high-grade, compared with low-grade, tumors $(12,18)$. Grade III-IV gliomas with high Ki-67 expression showed relatively low $\mathrm{k}_{3}$ values estimated by the Patlak approach, and this was associated with limited IDO expression mostly confined to endothelial cells. In contrast, low-grade brain tumors showed widespread IDO expression, consistent with high metabolic rates of AMT. Widespread expression of IDO in low-grade brain tumors may play a role in limiting proliferative activity via local tryptophan depletion while creating a tumor environment allowing long-term immune escape. In the present study, it was not possible to compare AMT kinetic variables across tumor grades or between various tumor types statistically. Nevertheless, both transport and metabolic rates were high in NSCLCs, consistent with a massive presence of IDO and the LAT-1 transporter as shown by previous studies $(3,9,13,32)$. This finding supports the notion that NSCLCs could be a good target for IDO inhibitors, and AMT PET could inform such trials. Other extracerebral malignancies known to express IDO or LAT-1 could be tested for a similar use of dynamic PET with AMT.

Our data show that AMT kinetics were not homogeneous, even within the NSCLC group: A few lesions showed a time-activity curve with an early peak, followed by a decline in activity within a few minutes after tracer injection, although retention was higher than that in corresponding lung tissue. Also, different tracer kinetics for each tumor could be seen even within a patient with multiple NSCLC lesions. Further AMT PET studies with a larger sample and comparisons with tissue findings, including measures of LAT transporters, IDO, or metabolites of the kynurenine pathway, could determine the underlying mechanisms of these differences in uptake. AMT PET time-activity curves often showed evidence of late efflux of the tracer or its metabolites from the tumor tissue (Fig. 2A). Although we did not calculate efflux rates, the signs of tracer efflux - which was particularly prominent and early in 3 cases of NSCLC (Fig. 2C) - raise the possibility of an effect of drug efflux transporters, which can contribute to drug resistance in a wide variety of malignancies, including lung cancers (34). How these transporters would affect retention of AMT in tumor tissue deserves further studies. Finally, a recent study demonstrated that tryptophan influx into cancer cells is tightly coupled with the efflux of kynurenine metabolites (35). This exchange process, performed by LAT transporters, facilitates tryptophan flux toward IDO-producing cells and protects these cells from kynurenine accumulation, thus diminishing the apoptotic effect of kynurenines. Considering this process, it is likely that tryptophan transport into IDO-expressing cells (estimated by $\mathrm{K}_{1}$ on AMT PET) and metabolite efflux (estimated by $\mathrm{k}_{4}$ ) are interrelated. Therefore, calculation of $\mathrm{K}_{1}$ 
(transport rate) and $\mathrm{k}_{3}$ (metabolic rate) in the simplified model provides an accurate characterization of kinetic movement of tryptophan in tumor tissue; determination of $\mathrm{k}_{4}$ may not yield substantial additional information in most cases while greatly diminishing the identifiability of the other parameters.

\section{CONCLUSION}

This initial study with dynamic PET/CT and noninvasively derived arterial blood input function in lung tumors demonstrates increased transport and metabolism of the tryptophan tracer AMT in NSCLCs, in which high AMT kinetic values are seen in tumors with high proliferative activity as suggested by high glucose uptake. Because abnormal tumor tryptophan metabolism via the kynurenine pathway is strongly implicated in tumor immunoresistance, AMT PET/CT may be a clinically useful molecular imaging method in identifying patients for pharmacologic targeting of tryptophan metabolism and monitoring treatment effects during emerging clinical trials with inhibitors of IDO and other therapeutic approaches targeting tryptophan transport or metabolism. PET of AMT kinetics could be also useful to further study whether increased IDO can facilitate or inhibit tumor growth in various extracerebral tumors.

\section{ACKNOWLEDGMENTS}

We thank Galina Rabkin, Angela Wigeluk, and Mei-li Lee for their technical assistance in performing the PET studies. This project has been funded in part with federal funds from the National Cancer Institute, National Institutes of Health, contract NO1-CO-12400. The content of this publication does not necessarily reflect the views or policies of the Department of Health and Human Services, nor does mention of trade names, commercial products, or organizations imply endorsement by the U.S. government. The study was also supported by a grant from the National Cancer Institute (CA-12341).

\section{REFERENCES}

1. Peters JC. Tryptophan nutrition and metabolism: an overview. Adv Exp Med Biol. 1991;294:345-358.

2. Schwarcz R, Pellicciari R. Manipulation of brain kynurenines: glial targets, neuronal effects, and clinical opportunities. J Pharmacol Exp Ther. 2002;303:1-10.

3. Uyttenhove C, Pilotte L, Theate I, et al. Evidence for a tumoral immune resistance mechanism based on tryptophan degradation by indoleamine 2,3dioxygenase. Nat Med. 2003;9:1269-1274.

4. Munn DH, Mellor AL. IDO and tolerance to tumors. Trends Mol Med. 2004;10:15-18.

5. Ozaki Y, Edelstein MP, Duch DS. Induction of indoleamine 2,3-dioxygenase: a mechanism of the antitumor activity of interferon gamma. Proc Natl Acad Sci USA. 1988;85:1242-1246.

6. Mellor AL, Munn DH. Tryptophan catabolism and T-cell tolerance: immunosuppression by starvation? Immunol Today. 1999;20:469-473.

7. Friberg M, Jennings R, Alsarraj M, et al. Indoleamine 2,3-dioxygenase contributes to tumor cell evasion of T cell-mediated rejection. Int J Cancer. 2002;101:151-155.

8. Travers MT, Gow IF, Barber MC, Thomson J, Shennan DB. Indoleamine 2,3dioxygenase activity and L-tryptophan transport in human breast cancer cells. Biochim Biophys Acta. 2004;1661:106-112.

9. Astigiano S, Morandi B, Costa R, et al. Eosinophil granulocytes account for indoleamine 2,3-dioxygenase-mediated immune escape in human non-small cell lung cancer. Neoplasia. 2005;7:390-396.
10. Okamoto A, Nikaido T, Ochiai K, et al. Indoleamine 2,3-dioxygenase serves as a marker of poor prognosis in gene expression profiles of serous ovarian cancer cells. Clin Cancer Res. 2005;11:6030-6039.

11. Brandacher G, Perathoner A, Ladurner R, et al. Prognostic value of indoleamine 2,3-dioxygenase expression in colorectal cancer: effect on tumor-infiltrating T cells. Clin Cancer Res. 2006;12:1144-1151.

12. Batista CEA, Juhász C, Muzik O, et al. Imaging correlates of differential expression of indoleamine 2,3-dioxygenase in human brain tumors. Mol Imaging Biol. In press.

13. Karanikas V, Zamanakou M, Kerenidi T, et al. Indoleamine 2,3-dioxygenase (IDO) expression in lung cancer. Cancer Biol Ther. 2007;6:1258-1262.

14. Ino K, Yamamoto E, Shibata K, et al. Inverse correlation between tumoral indoleamine 2,3-dioxygenase expression and tumor-infiltrating lymphocytes in endometrial cancer: its association with disease progression and survival. Clin Cancer Res. 2008;14:2310-2317.

15. Tohyama Y, Takahashi S, Merid MF, Watanabe A, Diksic M. The inhibition of tryptophan hydroxylase, not protein synthesis, reduces the brain trapping of alphamethyl-L-tryptophan: an autoradiographic study. Neurochem Int. 2002;40:603-610.

16. Shimizu T, Nomiyama S, Hirata F, Hayaishi O. Indoleamine 2,3-dioxygenase: purification and some properties. J Biol Chem. 1978;253:4700-4706.

17. Chugani DC, Muzik O. $\alpha$-[C-11]methyl-L-tryptophan PET maps brain serotonin synthesis and kynurenine pathway metabolism. J Cereb Blood Flow Metab. 2000;20:2-9.

18. Juhász $\mathrm{C}$, Chugani DC, Muzik $\mathrm{O}$, et al. In vivo uptake and metabolism of $\alpha-\left[{ }^{11} \mathrm{C}\right]$ methyl-L-tryptophan in human brain tumors. J Cereb Blood Flow Metab. 2006;26:345-357.

19. Patlak CS, Blasberg RG, Fenstermacher JD. Graphical evaluation of blood-tobrain transfer constants from multiple-time uptake data. J Cereb Blood Flow Metab. 1983;3:1-7.

20. Hamacher K, Coennen HH, Stoecklin G. Efficient stereospecific synthesis of nocarrier-added 2-[F-18]-fluoro-2-deoxy-D-glucose using aminopolyether supported nucleophilic substitution. J Nucl Med. 1986;27:235-238.

21. Chakraborty PK, Mangner TJ, Chugani DC, Muzik O, Chugani HT. A high-yield and simplified procedure for the synthesis of $\alpha-\left[{ }^{11} \mathrm{C}\right]$ methyl-L-tryptophan. Nucl Med Biol. 1996;23:1005-1008.

22. Muzik O, Behrendt DB, Mangner TJ, Chugani HT. Design of a protocol for quantitative brain FDG studies with PET not requiring invasive blood sampling [abstract]. J Nucl Med. 1994;35(suppl):104P.

23. Suhonen-Polvi H, Ruotsalainen U, Kinnala A, et al. FDG-PET in early infancy: simplified quantification methods to measure cerebral glucose utilization. $\mathrm{J} \mathrm{Nucl}$ Med. 1995;36:1249-1254.

24. Belsley D. Conditioning Diagnostics: Collinearity and Weak Data in Regression. New York, NY: Wiley \& Sons; 1991.

25. Chugani DC, Muzik O, Chakraborty P, Mangner TJ, Chugani HT. Human brain serotonin synthesis capacity measured in vivo with $\alpha$-[C-11]methyl-L-tryptophan. Synapse. 1998;28:33-43.

26. Vesselle H, Schmidt RA, Pugsley JM, et al. Lung cancer proliferation correlates with [F-18]fluorodeoxyglucose uptake by positron emission tomography. Clin Cancer Res. 2000;6:3837-3844.

27. Yamamoto Y, Nishiyama Y, Ishikawa S, et al. Correlation of ${ }^{18} \mathrm{~F}$-FLT and ${ }^{18} \mathrm{~F}-$ FDG uptake on PET with Ki-67 immunohistochemistry in non-small cell lung cancer. Eur J Nucl Med Mol Imaging. 2007;34:1610-1616.

28. Verrey F. System L: heteromeric exchangers of large, neutral amino acids involved in directional transport. Pflugers Arch. 2003;445:529-533.

29. Kanai Y, Segawa H, Miyamoto K, Uchino H, Takeda E, Endou H. Expression cloning and characterization of a transporter for large neutral amino acids activated by the heavy chain of 4F2 antigen (CD98). J Biol Chem. 1998;273:23629-23632.

30. Yanagida O, Kanai Y, Chairoungdua A, et al. Human L-type amino acid transporter 1 (LAT1): characterization of function and expression in tumor cell lines. Biochim Biophys Acta. 2001;1514:291-302.

31. Lin J, Raoof DA, Thomas DG, et al. L-type amino acid transporter-1 overexpression and melphalan sensitivity in Barrett's adenocarcinoma. Neoplasia. 2004;6:74-84.

32. Kaira K, Oriuchi N, Imai H, et al. Prognostic significance of L-type amino acid transporter 1 expression in resectable stage I-III nonsmall cell lung cancer. $\mathrm{Br} \mathrm{J}$ Cancer. 2008;98:742-748.

33. Kudo Y, Boyd CA. The role of L-tryptophan transport in L-tryptophan degradation by indoleamine 2,3-dioxygenase in human placental explants. J Physiol. 2001;531: 417-423.

34. Modok S, Mellor HR, Callaghan R. Modulation of multidrug resistance efflux pump activity to overcome chemoresistance in cancer. Curr Opin Pharmacol. 2006;6:350-354.

35. Kaper T, Looger LL, Takanaga H, Platten M, Steinman L, Frommer WB. Nanosensor detection of an immunoregulatory tryptophan influx/kynurenine efflux cycle. PLoS Biol. 2007;5:e257. 